\title{
Nanocomposites Based on PLLA and Multi Walled Carbon Nanotubes Support the Myogenic Differentiation of Murine Myoblast Cell Line
}

\author{
Simona Montesano, ${ }^{1}$ Erlantz Lizundia, ${ }^{2}$ Francesco D'Angelo, ${ }^{1}$ \\ Elena Fortunati, ${ }^{3}$ Samantha Mattioli, ${ }^{3}$ Francesco Morena, ${ }^{1}$ Ilaria Bicchi, ${ }^{1}$ Fabio Naro, ${ }^{4}$ \\ Maurilio Sampaolesi, ${ }^{5,6}$ Josè Ramon Sarasua, ${ }^{2}$ Josè Maria Kenny, ${ }^{3,7}$ Aldo Orlacchio, ${ }^{1}$ \\ Ilaria Armentano, ${ }^{3}$ and Sabata Martino ${ }^{1}$ \\ ${ }^{1}$ Department of Experimental Medicine and Biochemical Science, Section of Biochemistry and Molecular Biology, University of Perugia, \\ Via del Giochetto, 06126 Perugia, Italy \\ ${ }^{2}$ Department of Mining-Metallurgy and Materials Science, School of Engineering, University of the Basque Country (UPV/EHU), Spain \\ ${ }^{3}$ Materials Engineering Centre, UdR INSTM, NIPLAB, University of Perugia, Pentima Street 4, 05100 Terni, Italy \\ ${ }^{4}$ University of Roma-La Sapienza, 00100 Roma, Italy \\ ${ }^{5}$ Translational Cardiomyology Lab, Department of Development and Regeneration, SCIL, University of Leuven, 3000 Leuven, Belgium \\ ${ }^{6}$ Department of Public Health, Neuroscience, Experimental and Forensic Medicine and Center for Tissue Engineering (CIT), \\ University of Pavia, 27100 Pavia, Italy \\ ${ }^{7}$ Institute of Polymer Science and Technology (ICTP), CSIC, Calle de Juan de la Cierva 3, 28006 Madrid, Spain
}

Correspondence should be addressed to Ilaria Armentano; ilaria.armentano@unipg.it and Sabata Martino; martinos@unipg.it

Received 18 April 2013; Accepted 26 May 2013

Academic Editors: C. Haasper, J. Mansbridge, and Y.-J. Ren

Copyright (C) 2013 Simona Montesano et al. This is an open access article distributed under the Creative Commons Attribution License, which permits unrestricted use, distribution, and reproduction in any medium, provided the original work is properly cited.

We explored the effect of poly(L-lactic acid) (PLLA) containing various percentages $(0.1,0.5$, 1 , and 3 wt.\%) of multi walled carbon nanotubes (MWCNTs) on the myogenic differentiation of $\mathrm{C} 2 \mathrm{C} 12$ murine myoblast progenitor cells. We showed that all PLLA/MWCNTs nanocomposite materials support the myotubes formation more efficiently than neat PLLA as indicated by the high expression of the most significant myogenic markers: MyoD, Myosin Heavy Chain, dimension of myofibres, and fusion myogenic index. Interestingly, we note that both MyoD and myogenic fusion index levels were in the order $0.1 \mathrm{MWCNTs}=$ 0.5 MWCNTs $>1$ MWCNTs $>3$ MWCNTs $>$ neat PLLA, suggesting that the amount of MWCNTs influenced the cell differentiation.

\section{Introduction}

The goal of regenerative medicine is the generation of a biohybrid organ culture that closely mimics the physiological tissue developmental conditions through the combination of smart biomaterials and stem/precursor cells that could re-establish the metabolic alteration $[1,2]$. It is hypothesised that stem/ precursor cells could be able to convert the mechanical cues of biomaterials on biochemical signals and in turn modulate their fate. This phenomenon called mechanotransduction is under investigation intensively [2-4]. In this regard we have evaluated the interaction of stem cells and biomaterials showing that stem cells can act as mechanosensitive units responding to the material characteristic specifically. Thus adult, embryonic and induced pluripotent stem cells respond to nanocomposite poly(L-lactic acid) (PLLA) fibrous mats containing $1 \mathrm{wt}$.\% to $8 \mathrm{wt}$ \% amounts of calcium deficient hydroxyapatite with osteogenic differentiation [5], whereas human bone marrow mesenchymal stem cells (hBM-MSCs) respond to hydrogenated amorphous carbon with groove surface nanotopography with acquisition of neuronal characteristics [6]. 
Recently in our laboratory we have successfully developed and characterized the conductive nanocomposite films based on PLLA containing various percents $(0.1,0.5,1,3 \mathrm{wt} . \%)$ of multi walled carbon nanotubes (MWCNTs) (namely PLLA/MWCNTs: 0.1 MWCNTs, 0.5 MWCNTs, 1 MWCNTs, and 3 MWCNTs) [7].

PLLA is a biocompatible polymer matrix in nanocomposite production characterized by peculiar mechanical properties, adjustable degradation rate, and ease of manufacturing $[8,9]$. Carbon nanotubes are considered perfect reinforcing agents for high-strength and conductive polymer composites, because of their mechanical response and high electrical conductivity [10]. Thus PLLA/MWCNTs nanocomposites provide the possibility to modulate both electrical conductivity and mechanical properties $[10,11]$. In particular, electrical conductivity of PLLA/MWCNTs at micro- and nanoscale can influence the communication between cells and polymer nanocomposites [7, 12]. Finally, we have showed that PLLA/ MWCNTs support the culture and differentiation of hBMMSCs independently of the $(0.1,0.5,1$, and 3 wt.\%) MWCNTs content [7].

Here we further explored the effect of PLLA/MWCNTs on the cell biology. We selected the myogenic differentiation due to the potential of MWCNTs as electrical conductors. Experiments were performed culturing on each substrate murine $\mathrm{C} 2 \mathrm{C} 12$ myoblasts, skeletal muscle precursor cells that are usually referred as a myogenic cell model $[13,14]$. Reference studies were conducted culturing cells on neat PLLA.

Our results showed that PLLA/MWCNTs are suitable substrates for the generation of differentiated myofibers from progenitors $\mathrm{C} 2 \mathrm{C} 12$.

\section{Materials and Methods}

2.1. PLLA and PLLA/MWCNTs Process and Characterization. Nanocomposite films were prepared by solvent casting method in chloroform $\left(\mathrm{CHCl}_{3}\right)$, as previously described [6]. MWCNTs were dispersed in $\mathrm{CHCl}_{3}$ by using the tip sonicator (VIBRA CELL Sonics mod. VC 750, USA) for $30 \mathrm{~min}$ in ice bath. PLLA was mixed with nanotube dispersions in $\mathrm{CHCl}_{3}(10 \% \mathrm{wt} / \mathrm{v})$ by magnetic stirring for $5 \mathrm{~h}$ and, after it was completely dissolved, the mixture was cast on to a Teflon substrate and air dried at room temperature (RT) for $24 \mathrm{~h}$ and for a further $48 \mathrm{~h}$ in vacuum. Films of $60 \mathrm{~mm}$ in diameter and $0.2 \mathrm{~mm}$ thick were obtained. PLLA/MWCNTs samples containing $0.1,0.5,1$, and 3 wt. $\%$ as respect to PLLA initial weight and designed as $0.1 \mathrm{MWCNTs,} 0.5 \mathrm{MWCNTs}, 1 \mathrm{MWCNTs}$, 3 MWCNTs, respectively, were prepared. Neat PLLA film was also obtained by solvent casting for comparison.

The carbon nanotube dispersion within the PLLA matrix was evaluated using a transmission electron microscope (TEM, Philips CM120 Biofilter) at an acceleration voltage of $120 \mathrm{kV}$. Ultrathin sections of the nanocomposites (thickness of approx. $150 \mathrm{~nm}$ ) were cut using a Leica Ultracut UCT (LKB Ultratome III) ultramicrotome equipped with a diamond knife.

Surface morphologies of PLLA and PLLA/MWCNTs samples were studied with Veeco Instruments MultiMode
SPM 004-130-000 atomic force microscope (AFM) at RT, in contact mode. $\mathrm{A} \mathrm{Si}_{3} \mathrm{~N}_{4}$ cantilever (NP-10) with a characteristic force constant of approximately $0.58 \mathrm{~N} / \mathrm{m}$ and tip radius of $10 \mathrm{~nm}$ was employed. Scan rate of images was $1 \mathrm{~Hz}$ with a resolution of $256 \times 256$ data points per image.

2.2. Murine Myoblasts Culture and Differentiation. Murine myoblasts $\mathrm{C} 2 \mathrm{C} 12$ cells were seeded in $25 \mathrm{~cm}^{2}$ culture flasks at a density of $2.5 \times 10^{6}$ cells $/ \mathrm{mL}$ in control medium consisting of DMEM (EuroClone) medium containing 10\% heatinactivated fetal bovine serum (FBS), $2 \mathrm{mM}$ of L-glutamine, and $100 \mathrm{U} / \mathrm{mL}$ of penicillin-streptomycin (EuroClone) in a humidified atmosphere and 5\% carbon dioxide $\left(\mathrm{CO}_{2}\right)$ at $37^{\circ} \mathrm{C}$. The medium was changed every 2 days.

For $\mathrm{C} 2 \mathrm{C} 12$ differentiation experiments, the serum content in the growth media was decreased to $2 \%$ after 24 hours following seeding and maintained in culture for at least two weeks $[15,16]$.

2.3. Murine Myoblasts Culture and Differentiation on PLLA and PLLA/MWCNTs. Neat PLLA, 0.1MWCNTs, 0.5 MWCNTs, 1 MWCNTs, and 3 MWCNTs $(1 \mathrm{~cm} \times 1 \mathrm{~cm}$ each), were disinfected through immersion in $70 \%$ ethanol for $30 \mathrm{sec}$ followed by three rinses in phosphate-buffer saline (PBS) and then deposited in a 24 -well plate. $50 \mu \mathrm{L}$ of C2C12 suspension (500 cells) were seeded dropwise, on those substrates. $500 \mu \mathrm{L}$ of culture medium consisting of DMEM (EuroClone) medium containing 10\% heat-inactivated fetal bovine serum (FBS), $2 \mathrm{mM}$ of L-glutamine, and $100 \mathrm{U} / \mathrm{mL}$ of penicillin-streptomycin was added to the appropriate wells. The medium was changed every 3 days. Myogenic differentiation of $\mathrm{C} 2 \mathrm{C} 12$ was achieved by decreasing to $2 \%$ the FBS in the growth media after $24 \mathrm{~h}$ following seeding on each material. Culture conditions were monitored for 14 days according to published protocols $[15,16]$. Myotubes formation was evaluated by specific myogenic markers.

2.4. Cell Viability Assay. At different time points (3, 7, and 14 days), cell viability was measured by assaying the mitochondrial dehydrogenase activity by incubating cultures with XTT salt solution (Sigma Aldrich) for $4 \mathrm{~h}$ at $37^{\circ} \mathrm{C}$ according to the manufacturer's recommendations. The absorbance of the samples was measured using a microtiter plate reader (GDV) at $450 \mathrm{~nm}$ with a reference wavelength at $650 \mathrm{~nm}$.

2.5. Immunofluorescence. Immunofluorescence was performed as already described [17]. Briefly, cells were fixed in $4 \%$ paraformaldehyde for $30 \mathrm{~min}$, permeabilized, and blocked (PBS $+10 \%$ FBS, $0.1 \%$ Triton X-100) for $1 \mathrm{~h}$ at RT, and when occurred incubated with phalloidin (AlexaFluor $488 \mathrm{~nm}$ phalloidin, Invitrogen), for $20 \mathrm{~min}$, and then further incubated overnight at $4^{\circ} \mathrm{C}$ with primary antibodies: anti-alpha-tubulin (Santa Cruz Biotechnology, Santa Cruz, CA), anti-MHC and anti-MyoD were generated in our laboratories. Finally, after being washed with PBS and stained with Alexa-Fluor $594 \mathrm{~nm}$ conjugated secondary antibodies (Invitrogen) for $1 \mathrm{~h}$ at RT, samples were mounted, and nuclei were counterstained with Vectashield with DAPI 


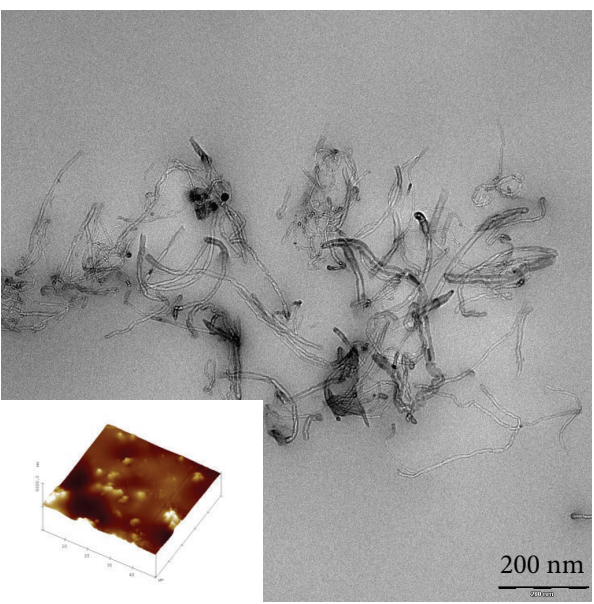

(a)

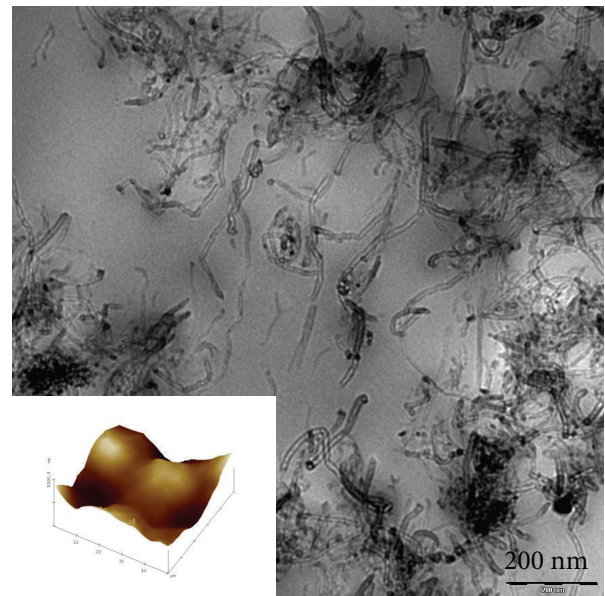

(b)

Figure 1: PLLA and PLLA/MWCNTs nanocomposite films. TEM micrographs showing randomly distributed carbon nanotubes for 0.1 MWCNTs (a) and 3 MWCNTs (b) nanocomposites. The inserts show the contact-mode 3D AFM images for the same compositions.

(Vector Laboratories Inc.). Images were acquired using fluorescence microscopy (Eclipse-TE2000-S, Nikon) using the F-View-II FireWire camera (Soft Imaging System, NIS, Nikon) and elaborated using the Adobe Photoshop CS4 program.

2.6. Elongation Factor. To evaluate cell elongation factors $(E)$, measurements were performed on days 3, 7, and 14 of culture for each substrate (PLLA, 0.1 MWCNTs, 0.5 MWCNTs, $1 \mathrm{MWCNTs}$, and $3 \mathrm{MWCNTs})$. Eight different areas were photographed (20x magnification). The $E$ factor is defined as the ratio between long and short axis minus 1 . Thus, $E=0$ for a circle, and $E=1$ for an ellipse with an axis ratio of 0.5 $[6,17,18]$. For each condition an average of 300 cells were analyzed. Images were analysed as described before.

2.7. Myotubes Characterization. Expression of the muscle differentiation transcription factor MyoD was calculated by counting the MyoD positive nuclei in a given immunofluorescence image $(20 x$, more than six images for each sample and the total number of nuclei analyzed was 300-500/field). The MyoD negative nuclei were also counted.

A myotube is defined as a cell containing two or more nuclei [19]. Myotubes characterization measurements, width and length, were analyzed at 14 days by staining of myosin heavy chain (MHC) positive fibres from microscopic images (20x, more than six images for each sample; the number of myotubes $>70$ ). The myoblast fusion, or myogenic index, is defined as the number of nuclei residing in cells containing three or more nuclei divided by the total number of nuclei in a given image [20]. Nuclei were counted from microscopic images taken from eight different areas (20x magnification; six images for each sample were considered). Total number of nuclei analyzed was 300-500/field.

2.8. Statistical Analysis. Data are reported as the mean value \pm mean standard error (SEM). Quantitative data were compared using the unpaired Student's $t$-test $(P \leq 0.05)$.
Prism version 4 (2004 edition; GraphPad, La Jolla, CA) was used for statistical analyses.

\section{Results}

3.1. PLLA and PLLA/MWCNTs Characterization. Neat PLLA and PLLA containing 0.1, 0.5, 1, and 3 wt.\%. MWCNTs were successfully fabricated as described in experimental section and characterized as previously reported [7].

Here we reported the PLLA/MWCNTs properties that could be relevant for the analysis of cell behaviour.

TEM micrographs confirmed the randomly distribution of nanotubes within the whole PLLA matrix for all the composition studied. In fact, at low concentrations, nanotubes appear as separated individual tubes (Figure 1(a)). However, as the nanotube concentration increases, MWCNTs form an interconnected structure, which gives rise to entanglements among adjacent MWCNTs, resulting in formation of nanotube aggregates for concentrations higher than $1 \mathrm{wt} . \%$, as 3 MWCNTs (Figure $1(\mathrm{~b})$ ). It is worth to note that the transition from well-dispersed distribution of MWCNTs to aggregate structure is produced for a composition composed of between 1.25 and 2.5 wt.\% MWCNTs; while a conductive behavior was found for MWCNT contents larger than $0.25 \mathrm{wt} \%$, composites with MWCNT content lower than or equal to $0.1 \mathrm{wt} \%$ show an insulating character [7]. In addition, AFM studies were conducted to analyse the uniformity of dispersions of the filler as well as for the characterization of the surface morphology of PLLA/MWCNT nanocomposites that would interact with $\mathrm{C} 2 \mathrm{C} 12$ cells. The inserts in Figure 1 shows the representative AFM 3D surface topology of the samples containing 0.1 (A) and 3 (B) wt.\% MWCNTs. By means of adding nanotubes to the polymer, highest aggregation of MWCNT is found generating irregularities with no preferential orientation on the surface, providing isotropic electrical and thermal properties to PLLA/MWCNTs nanocomposites [21]. It can be observed that surface remains almost flat with the addition of nanotubes up to 1.25 wt. $\%$. At higher loadings 


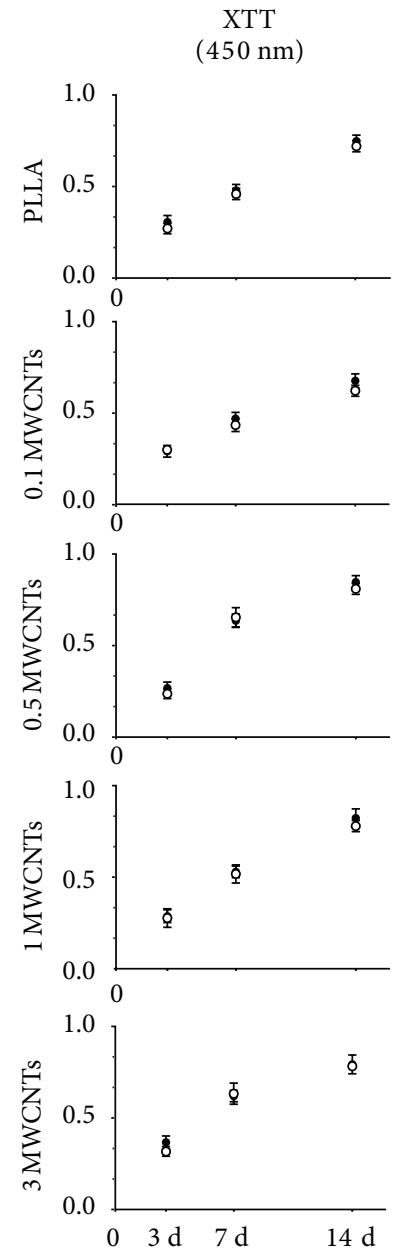

(a)

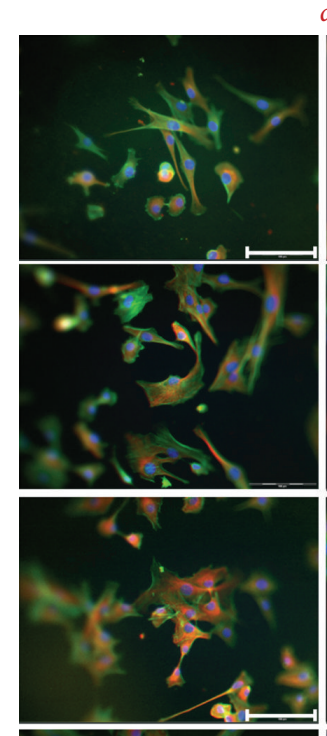

$\alpha$-Tubulin/F-actin/DAPI
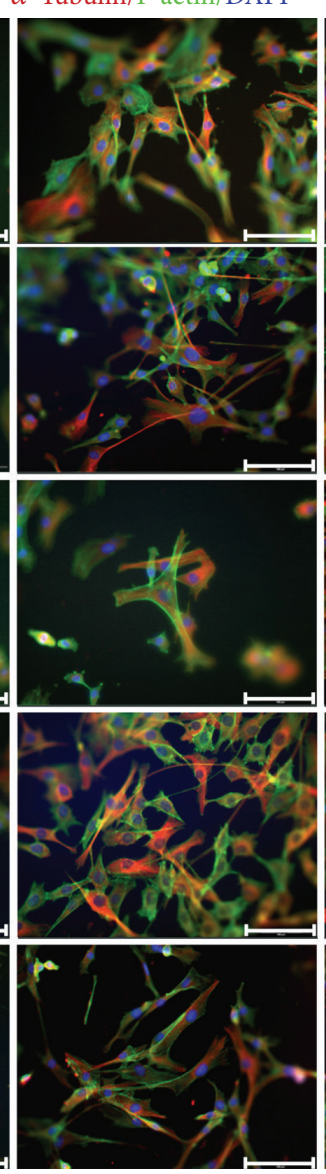

$7 \mathrm{~d}$

(b)

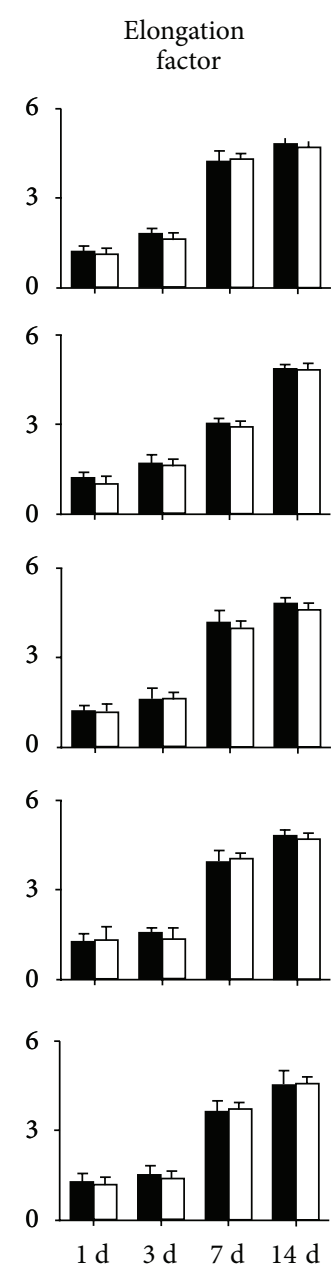

(c)

FIgure 2: Interaction of C2C12 with PLLA and PLLA/MWCNTs nanocomposite films. Murine C2C12 myoblasts were cultured on PLLA, 0.1 MWCNTs, 0.5 MWCNTs, 1 MWCNTs, and 3 MWCNTs. (a) XTT viability assay of C2C12 plated on different substrates, at 3, 7, and 14 days. Results were comparable for cells grown on smoothness $(\bullet$ ) and roughness (o) surfaces. (b) Representative images of C2C12 seeded on each substrate, at 3, 7, and 14 days. Microtubule organization is showed by $\alpha$-tubulin (tetramethylrhodamine isothiocyanate (TRIC))/Factin (fluorescein isothiocyanate (FITC)) $/ 4^{\prime}, 6$-diamidino-2-phenylindole (DAPI) staining. Images were captured with 20x objectives; scale bar: $100 \mu \mathrm{m}$. (c) Elongation factor (11) was measured for cells cultured on each substrate, on smoothness ( $\square$ ) and roughness ( $\square$ ) surfaces, at different intervals of time $(1,3,7$, and 14 days). Each experiment consisted of at least five technical replicates. Values are expressed as means \pm $\operatorname{SEM}(P \leq 0.05)$.

MWCNTs yield a "mountain-valley" feature at micrometer scale whose valley depth ranges from $438 \mathrm{~nm}$ for nanocomposite containing $0.1 \mathrm{wt}$. $\%$ to $1526 \mathrm{~nm}$ for its $3 \mathrm{wt}$.\% nanocomposite. In addition, the mean roughness of neat PLLA is found to be about $53 \mathrm{~nm}$ comparing to $580 \mathrm{~nm}$ obtained for the 3 MWCNTs nanocomposite.

\subsection{C2C12 Cell Interaction with PLLA and PLLA/MWCNTs} Nanocomposites Films. For this set of experiments, C2C12 cells were cultured either on the smoothest (bottom side) or the roughness surfaces (upper side) of neat PLLA, 0.1 MWCNTs, 0.5 MWCNTs, 1 MWCNTs, and 3 MWCNTs (Figure 2).

First, we evaluated the cell viability measuring the mitochondrial dehydrogenate activity, at different time points
(3, 7, and 14 days) of C2C12 cultured on PLLA and PLLA/MWCNTs (Figure 2(a)). All cell patches showed comparable levels of mitochondrial dehydrogenase activity and absence of signs of toxicity such as appearance of cellular debris in the culture medium. Results were similar for cells grown on smoothest and roughness surfaces of nanocomposites and neat PLLA (Figure 2(a)).

Next, we analyzed the cell-material interaction by $\alpha$ tubulin/F-actin immunostaining. After 3 days of culture, the interaction of $\mathrm{C} 2 \mathrm{C} 12$ with neat PLLA, 0.1 MWCNTs, 0.5 MWCNTs, 1 MWCNTs, and 3 MWCNTs appeared as typical spindle cell morphology of myoblasts (Figure 2(b) column 3d). After 7 days of culture, the myoblasts started to become "thin-tapered" due to the increase of cell density (Figure 2(b), column 7d). At day 14, "end to end" contacts between "thin-taped myoblasts" were observed 

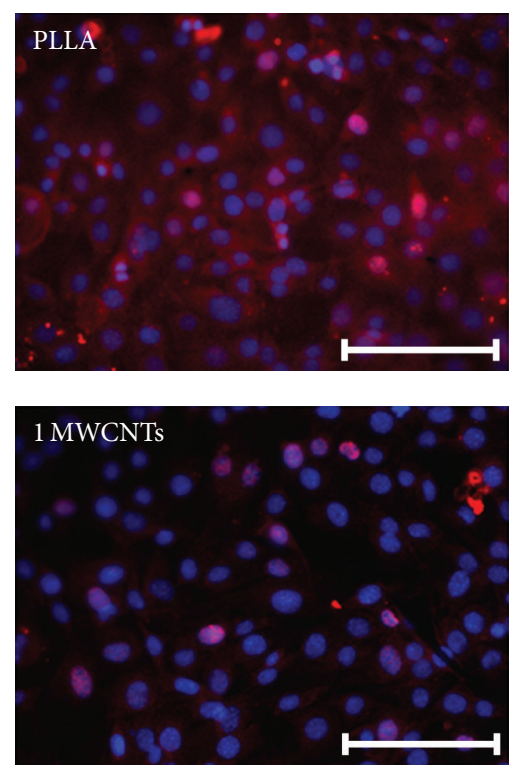

MyoD/DAPI
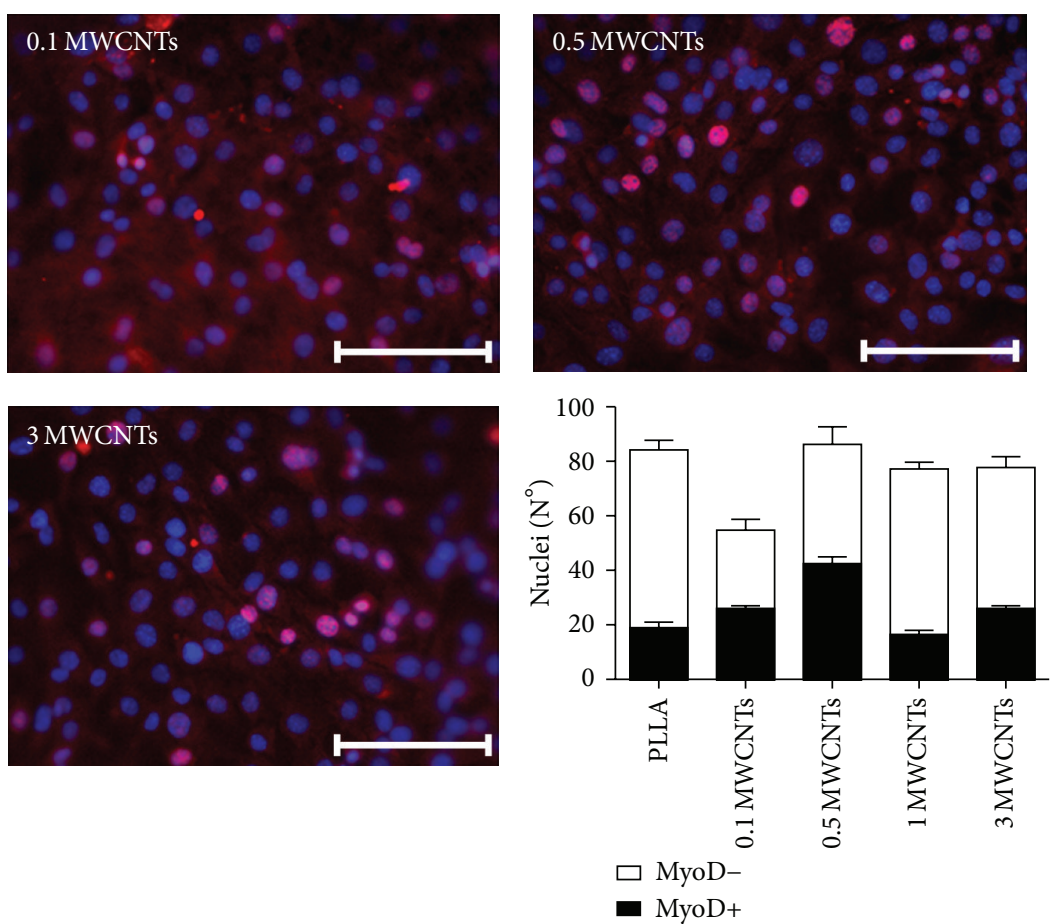

Figure 3: MyoD expression in C2C12 cultured on neat PLLA and PLLA/MWCNTs. Immunofluorescences of murine C2C12 cultured in 2\% FBS containing medium for 2 days express the early myogenic marker MyoD (RED) either in neat PLLA and PLLA/MWCNTs. Cell nuclei (BLUE) were stained with DAPI. Scale bar: $100 \mu \mathrm{m}$. Graph reports MyoD expression. Level is defined as the number of MyoD positive nuclei $(\mathbf{\square})$ in a given image and was calculated in differentiated cells cultivated on each substrate. As reference numbers of MyoD negative nuclei are reported $(\square)$. Each experiment consisted of at least five technical replicates. Values are expressed as means \pm SEM $(P \leq 0.05)$.

on neat PLLA, 0.1 MWCNTs, 0.5 MWCNTs, 1 MWCNTs, and 3 MWCNTs (Figure 2(b), column 14d). Morphological images were similar for $\mathrm{C} 2 \mathrm{C} 12$ seeded on both smoothest (Figure 2(b), representative images) and roughness surfaces (data not showed) in each substrate.

Transition from "tapered to thin-tapered" morphology during the culture period was confirmed by the measure of the cellular elongation factor (Figure 2(c)). We observed an increase of the elongation factor with time in culture that was almost identical for all substrates considered (Figure 2(c)). Similar results were obtained for cells grown on smoother or rougher surfaces (Figure 2(c)).

\subsection{Myogenic Differentiation of C2C12 Cells Seeded on PLLA} and PLLA/MWCNTs. In order to generate myotubes from C2C12, cells were seeded on neat PLLA and PLLA/MWCNTs and induced to differentiate in the presence of medium containing 2\% FBS for 14 days. The muscle differentiation was monitored evaluating the expression of the MyoD transcription factor as early myogenic marker and the expression of MHC as a late differentiation marker.

After 2 days of culture in differentiation medium, cells express MyoD (Figure 3, representative images) in the percentages of $19 \pm 2 \%, 49 \pm 4 \%, 50 \pm 3 \%, 28 \pm 3 \%$, and $31 \pm 1 \%$ for PLLA, 0.1 MWCNTs, 0.5 MWCNTs, 1 MWCNTs, and 3 MWCNTs, respectively (Figure 3, histogram).

After 14 days in differentiation medium, we noticed the presence of myotubes on neat PLLA, 0.1MWCNTs,
0.5 MWCNTs, 1 MWCNTs, and 3 MWCNTs (Figure 4, representative images). However, according to the MyoD expression, we observed differences in terms of number of myotubes in the order 0.1 MWCNTs $=0.5$ MWCNTs $>1$ MWCNTs > 3 MWCNTs > PLLA (data not shown). This trend was confirmed by the measurement of the fusion myogenic index (Figure 5(a)) and the length of myotubes: 0.1 MWCNTs = $490 \pm 20 \mu \mathrm{m} ; 0.5 \mathrm{MWCNTs}=460 \pm 40 \mu \mathrm{m} ; 1 \mathrm{MWCNTs}=$ $400 \pm 40 \mu \mathrm{m} ; 3 \mathrm{MWCNTs}=350 \pm 40 \mu \mathrm{m}$; neat PLLA $=$ $250 \pm 15 \mu \mathrm{m}$ (Figure 5(b)). Conversely the average width of myotubes on neat PLLA $(22 \pm 8 \mu \mathrm{m})$ was comparable with those measured on the nanocomposite materials $(0.1 \mathrm{MWCNTs}=23 \pm 6 \mu \mathrm{m} ; 0.5 \mathrm{MWCNTs}=19 \pm 4 \mu \mathrm{m}$; $1 \mathrm{MWCNTs}=20 \pm 4 \mu \mathrm{m} ; 3 \mathrm{MWCNTs}=19 \pm 4 \mu \mathrm{m}$ ) on day 14 (Figure 5(c)).

Together these data indicated that PLLA/MWCNTs favour the differentiation of $\mathrm{C} 2 \mathrm{C} 12$ toward myotubes and suggest a potential correlation between MWCNTs content and differentiation.

\section{Discussion}

The current biotechnologies for the generation of artificial organs and regeneration of degenerated tissues are based on the development of hybrid systems consisting of a cellular source (organic component) and a biomaterial as functional support $[1-3,22]$. In this regard effort has been made on designing and fabricating materials able to drive the stem cell 


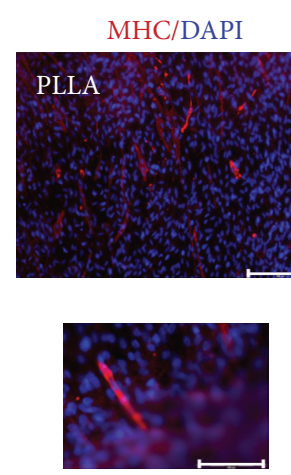

(a)
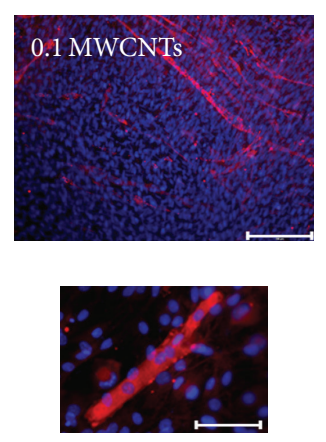

(b)
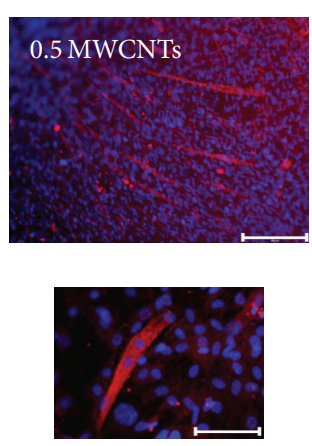

(c)
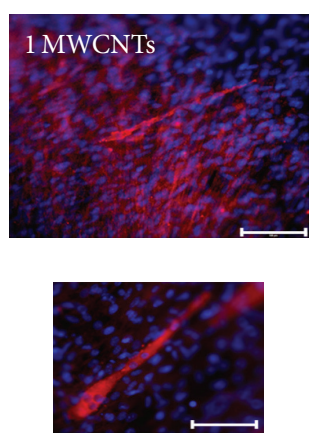

(d)
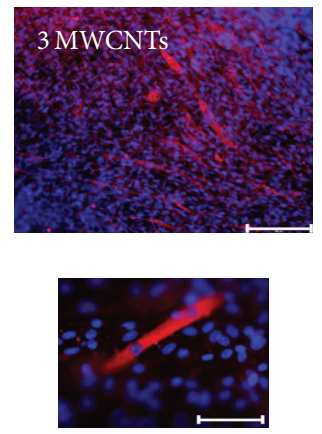

(e)

FIGURE 4: MHC expression and myotubes on neat PLLA and PLLA/MWCNTs. Immunofluorescence showed well-organized MHC protein (RED) in differentiated C2C12 on PLLA and PLLA/MWCNTs after 14 days of differentiation. Cell nuclei (blue) were stained with DAPI. Scale bar: $100 \mu \mathrm{m}$. ((a), (b), (c), (d), and (e)): myotubes, representative high magnification images of. PLLA, 0.1 MWCNTs, 0.5 MWCNTs, 1 MWCNTs, and 3 MWCNTs, respectively.

fate toward a specific lineage and on elucidating the molecular events by which cells respond to the material properties [1$3,22,23]$.

In this paper, we showed that the myogenic index is influenced by the content of multi walled carbon nanotubes.

Recently, we successfully produced PLLA nanocomposite films with carbon nanotubes by solvent casting. The characterization of the materials showed that MWCNTs improve the PLLA electrical conductivity at very low concentrations with a formation of a tridimensional nanotube network in the percolated formulations [7]. Analytical characterization by AFM showed an increasing surface roughness due to the presence of carbon nanotubes, yielding a continuous increase in surface hydrophilicity as the nanotube content increases.

Here we reported that PLLA/MWCNTs nanocomposites support myogenic cell cultures. In fact cell viability, morphological evaluation, and $\mathrm{C} 2 \mathrm{C} 12$ differentiation persist unaltered in neat PLLA and PLLA/MWCNTs nanocomposites. Notably, we observed the absence of toxic effects of MWCNTs, even at high percentages. These results were comparable for cells cultured in both sides of all substrates considered in our study.

Myoblast differentiation into myotubes depends on two critical events: cell cycle exit and end-to-end contacts between myoblasts [24]. Division and differentiation of myoblasts are reciprocally exclusive events controlled by a balance of two sets of opposing cellular signals. Escape of myoblasts from the cell cycle is necessary for induction of musclespecific transcription factors and many late myogenic genes [25].

The PLLA/MWCNTs nanocomposite materials support myotube formation without alteration of cell viability and entrance to the differentiated state. We observed high rate of expression of the early myogenic marker MyoD as well as the late differentiation marker MHC. Moreover, one of the crucial factors in the formation of skeletal muscle tissue, the dimension of myofibres, corresponded to the canonical dimensions of myogenic differentiated cells [20] as showed by width myotubes, which ranged from $250 \mu \mathrm{m}$ to $500 \mu \mathrm{m}$ in length, PLLA, and PLLA/MWCNTs, respectively. Remarkable MyoD and myogenic fusion index level were in the order $0.1 \mathrm{MWCNTs}=0.5 \mathrm{MWCNTs}>1 \mathrm{MWCNTs}>3 \mathrm{MWCNTs}>$ neat PLLA suggesting that the presence of MWCNTs influenced the efficiency of cell differentiation. It is likely that low content of MWCNTs ( $0.1 \mathrm{wt} . \%$ and $0.5 \mathrm{wt} . \%$ ) had an enhancing myogenic effect while this is strongly reduced by the MWCNTs increase (1 wt.\% and $3 \mathrm{wt} . \%)$. We speculated that the properties conferred by low MWCNTs content to the PLLA (e.g., level of surface roughness and a continuous increase in surface hydrophilicity as the nanotube content increase) generated a nanocomposite that is more myogenic specific.

Indeed, surface morphology, surface wettability, and size distribution of biomaterials could affect the in vitro and in vivo tissue regeneration potential $[26,27]$. Thus, in vivo and in vitro studies correlated the effect of surface morphology, surface wettability, mean size, and size distribution of biomaterial with the osteoblastic differentiation (upregulation of the expression of RUNX2 [osteogenic growth factor gene], alkaline phosphatase, osteonectin, and osteocalcin, calcium deposition) and the regeneration of new bone in rat [26]. Additionally, submicron scale roughness and hydrophilicity surface modifications on titanium substrates promote osteogenic cell adhesion and differentiation and highlight the healing of dental endosseous implants [28]. Finally, the effect of hydrophobicity and surface roughness of glass and zirconia/silica microbead cultivation system has been shown to influence the growth and the production of the antibiotic granaticin by Streptomyces granaticolor [27].

\section{Conclusion}

Altogether our results demonstrated that PLLA/MWCNTs nanocomposites are suitable substrates for the generation of differentiated myofibers from $\mathrm{C} 2 \mathrm{C} 12$ progenitor cells. Remarkably, the myogenic differentiation (in terms of MyoD and MHC expression and myogenic index) was highest on 


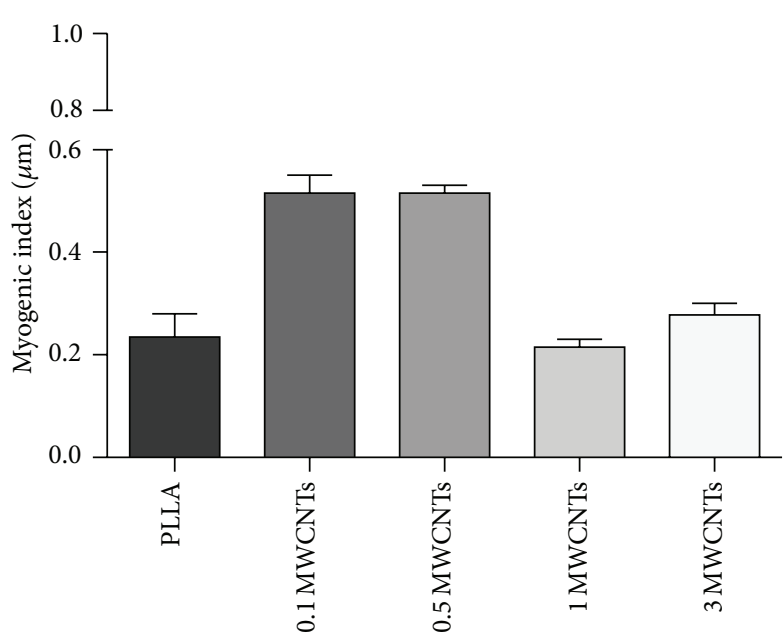

(a)

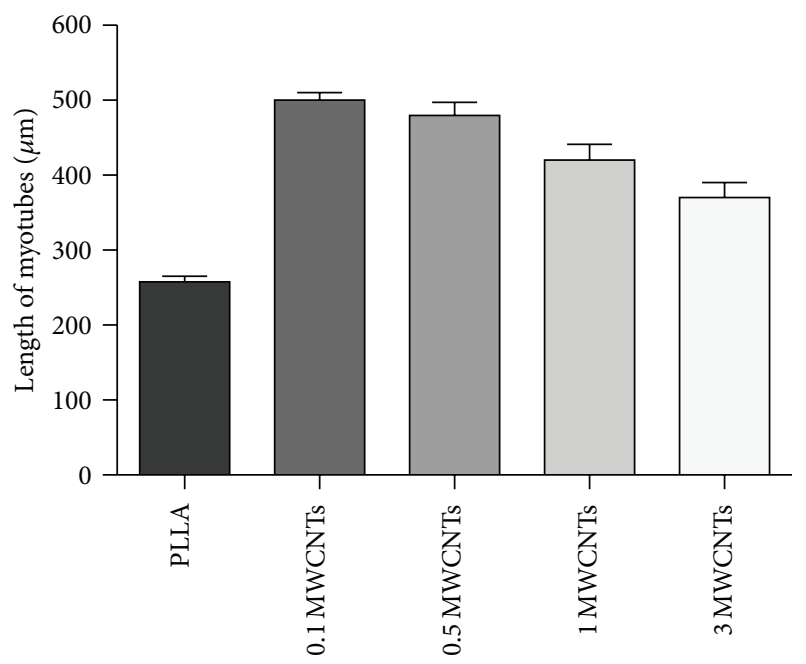

(b)

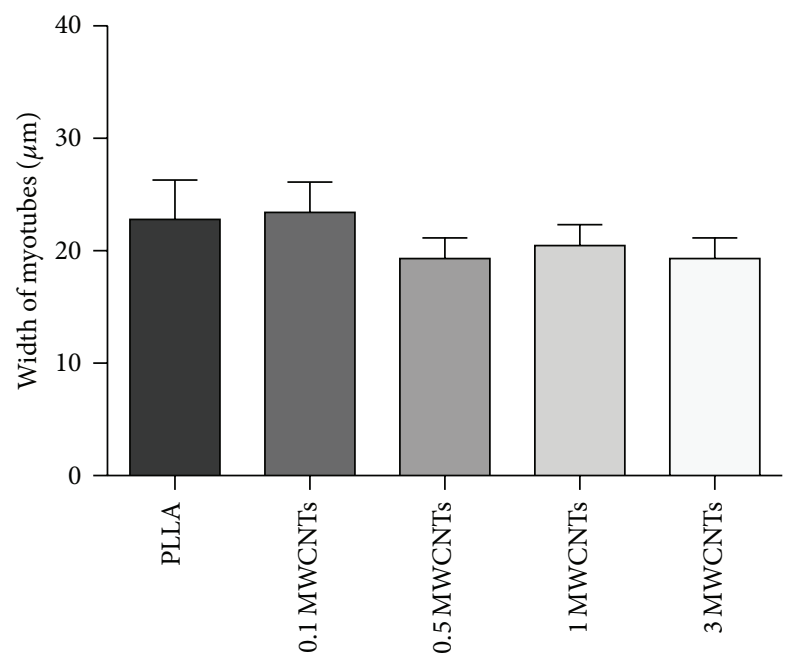

(c)

FIGURE 5: Myogenic parameters in C2C12 after differentiation on neat PLLA and PLLA/MWCNTs. (a) Myogenic index, defined as the number of 2-3 nuclei residing in myotubes divided by total number of nuclei in a given image, was calculated in differentiated cells cultivated on different substrates. Measurement of length (b) and width (c) of myotubes. Quantitative analysis of the morphology of myotubes after 14 days of differentiation was performed by using the fluorescence microscopy Eclipse-TE2000-S Nikon, equipped with the F-View-II FireWire camera and the Soft Imaging System NIS, Nikon. Average width and average length are reported. Each experiment consisted of at least five technical replicates. Values are expressed as means \pm SEM $(P \leq 0.05)$.

0.1 MWCNTs and 0.5 MWCNTs compared to 1 MWCNTs and $3 \mathrm{MWCNTs}$ indicating that nanocomposites properties influence the efficiency of the myogenic process. These observations were confirmed by the lowest myogenic differentiation monitored in $\mathrm{C} 2 \mathrm{C} 12$ cultured on neat PLLA.

More experiments are needed in order to explain the mechanisms but the overall results confirm that the interaction of cell and biomaterial is a crucial step for inducing the molecular events that drive the cell differentiation response.

\section{Acknowledgments}

This study was supported by the Italian Fondazione Cassa di Risparmio di Perugia (Grant no. 2010.011.0445 to Sabata
Martino) and the Istituto Nazionale Biostrutture e Biosistemi. Erlantz Lizundia thanks the University of Basque Country (UPV/EHU) for a postdoctoral fellowship. The Author Elena Fortunati is the recipient of the fellowship "L'Oreal Italia per le Donne e la Scienza 2012" for the project "Progettazione, sviluppo e caratterizzazione di biomateriali nanostrutturati capaci di modulare la risposta e il differenziamento delle cellule staminali." The authors thank Professor Nicoletta Francia (English Language Speaker) for the proofreading the paper.

\section{References}

[1] S. J. Lee and A. Atala, "Scaffold technologies for controlling cell behavior in tissue engineering," Biomedical Materials, vol. 8, no. 1, Article ID 010201, 2013. 
[2] B. Inanç and Y. M. Elçin, "Stem cells in tooth tissue regeneration-challenges and limitations," Stem Cell Reviews, vol. 7, no. 3, pp. 683-692, 2011.

[3] E. K. Yim and M. P. Sheetz, "Force-dependent cell signaling in stem cell differentiation," Stem Cell Research \& Therapy, vol. 3, no. 5 , article $41,2012$.

[4] S. Martino, F. D’Angelo, I. Armentano, J. M. Kenny, and A. Orlacchio, "Stem cell-biomaterial interactions for regenerative medicine," Biotechnology Advances, vol. 30, no. 1, pp. 338-351, 2012.

[5] F. D’Angelo, I. Armentano, I. Cacciotti et al., “Tuning multi/ pluri-potent stem cell fate by electrospun poly(L-lactic acid)calcium-deficient hydroxyapatite nanocomposite mats," Biomacromolecules, vol. 13, no. 5, pp. 1350-1360, 2012.

[6] F. D’Angelo, I. Armentano, S. Mattioli et al., "Micropatterned hydrogenated amorphous carbon guides mesenchymal stem cells towards neuronal differentiation," European Cells \& Materials, vol. 20, pp. 231-244, 2010.

[7] E. Lizundia, J. R. Sarasua, F. D’Angelo et al., "Biocompatible Poly (L-lactide) / MWCNT nanocomposites: morphological characterization, electrical properties and stem cell interaction," Macromolecular Bioscience, vol. 12, no. 7, pp. 870-881, 2012.

[8] A. Bianco, C. Del Gaudio, S. Baiguera et al., "Microstructure and cytocompatibility of electrospun nanocomposites based on poly( $\varepsilon$-caprolactone) and carbon nanostructures," International Journal of Artificial Organs, vol. 33, no. 5, pp. 271-282, 2010.

[9] M. Dottori, C. Tay, and S. M. Hughes, "Neural development in human embryonic stem cells-applications of lentiviral vectors," Journal of Cellular Biochemistry, vol. 112, no. 8, pp. 1955-1962, 2011.

[10] I. Armentano, L. Marinucci, M. Dottori et al., "Novel poly(Llactide) PLLA/SWNTs nanocomposites for biomedical applications: material characterization and biocompatibility evaluation," Journal of Biomaterials Science, Polymer Edition, vol. 22, no. 4-6, pp. 541-556, 2011.

[11] E. M. Cronin, F. A. Thurmond, R. Bassel-Duby et al., "Proteincoated poly(L-lactic acid) fibers provide a substrate for differentiation of human skeletal muscle cells," Journal of Biomedical Materials Research A, vol. 69, no. 3, pp. 373-381, 2004.

[12] S. Sun, I. Titushkin, and M. Cho, "Regulation of mesenchymal stem cell adhesion and orientation in 3D collagen scaffold by electrical stimulus," Bioelectrochemistry, vol. 69, no. 2, pp. 133$141,2006$.

[13] D. Yaffe and O. Saxel, "Serial passaging and differentiation of myogenic cells isolated from dystrophic mouse muscle," Nature, vol. 270, no. 5639, pp. 725-727, 1977.

[14] S. T. Cooper, A. L. Maxwell, E. Kizana et al., "C2C12 coculture on a fibroblast substratum enables sustained survival of contractile, highly differentiated myotubes with peripheral nuclei and adult fast myosin expression," Cell Motility and the Cytoskeleton, vol. 58, no. 3, pp. 200-211, 2004.

[15] A. Consiglio, S. Martino, D. Dolcetta et al., "Metabolic correction in oligodendrocytes derived from metachromatic leukodystrophy mouse model by using encapsulated recombinant myoblasts," Journal of the Neurological Sciences, vol. 255, no. 1-2, pp. 7-16, 2007.

[16] S. Martino, A. Consiglio, C. Cavalieri et al., "Expression and purification of a human, soluble Arylsulfatase a for Metachromatic Leukodystrophy enzyme replacement therapy," Journal of Biotechnology, vol. 117, no. 3, pp. 243-251, 2005.

[17] S. Martino, F. D’Angelo, I. Armentano et al., "Hydrogenated amorphous carbon nanopatterned film designs drive human bone marrow mesenchymal stem cell cytoskeleton architecture," Tissue Engineering A, vol. 15, no. 10, pp. 3139-3149, 2009.

[18] E. K. F. Yim and K. W. Leong, "Significance of synthetic nanostructures in dictating cellular response," Nanomedicine, vol. 1, no. 1, pp. 10-21, 2005.

[19] P.-Y. Wang, H.-T. Yu, and W.-B. Tsai, "Modulation of alignment and differentiation of skeletal myoblasts by submicron ridges/ grooves surface structure," Biotechnology and Bioengineering, vol. 106, no. 2, pp. 285-294, 2010.

[20] N. Wedhas, H. J. Klamut, C. Dogra, A. K. Srivastava, S. Mohan, and A. Kumar, "Inhibition of mechanosensitive cation channels inhibits myogenic differentiation by suppressing the expression of myogenic regulatory factors and capcase-3 activity," FASEB Journal, vol. 19, no. 14, pp. 1986-1997, 2005.

[21] E. Lizundia, A. Oleaga, A. Salazar, and J. R. Sarasua, "Nanoand microstructural effects on thermal properties of poly (1lactide)/multi-wall carbon nanotube composites," Polymer, vol. 53, no. 12, pp. 2412-2421, 2012.

[22] K. Kolind, K. W. Leong, and F. Besenbacher M, "Guidance of stem cell fate on 2D patterned surfaces," Biomaterials, vol. 33, no. 28, pp. 6626-6633, 2012.

[23] S. F. Badylak, D. J. Weiss, A. Caplan, and P. MacChiarini, "Engineered whole organs and complex tissues," The Lancet, vol. 379, no. 9819, pp. 943-952, 2012.

[24] P. Clark, G. A. Dunn, A. Knibbs, and M. Peckham, "Alignment of myoblasts on ultrafine gratings inhibits fusion in vitro," International Journal of Biochemistry and Cell Biology, vol. 34, no. 7, pp. 816-825, 2002.

[25] E. N. Olson, "Interplay between proliferation and differentiation within the myogenic lineage," Developmental Biology, vol. 154, no. 2, pp. 261-272, 1992.

[26] S. Tavakol, M. R. Nikpour, and A. Amani, "Bone regeneration based on nano-hydroxyapatite, and hydroxyapatite/chitosan nanocomposites: an in vitro, and in vivo comparative study," Journal of Nanoparticle Research, vol. 15, pp. 1373-1378, 2013.

[27] D. Petráčková, K. Buriánková, E. Tesařová et al., "Surface hydrophobicity and roughness influences the morphology and biochemistry of streptomycetes during attached growth and differentiation," FEMS Microbiology Letters, vol. 342, no. 2, pp. 147-156, 2013.

[28] M. O. Klein, A. Bijelic, T. Ziebart et al., "Submicron scale-structured hydrophilic titanium surfaces promote early osteogenic gene response for cell adhesion and cell differentiation," Clinical Implant Dentistry and Related Research, vol. 15, no. 2, pp. 166$175,2011$. 


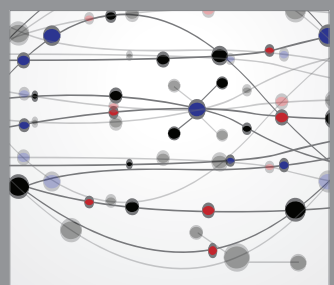

The Scientific World Journal
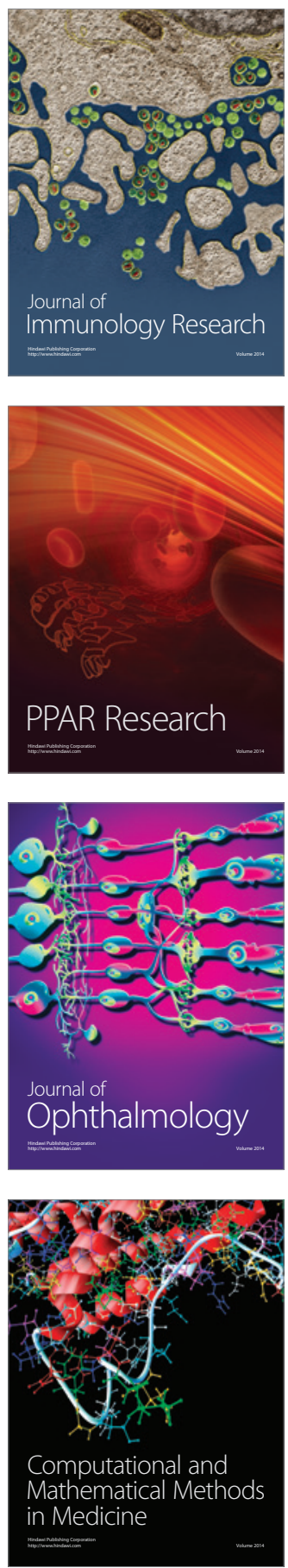

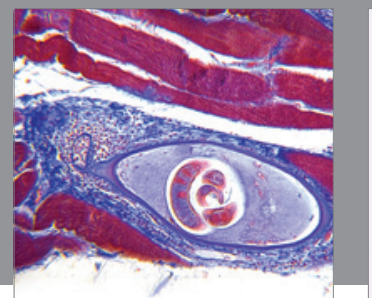

Gastroenterology

Research and Practice
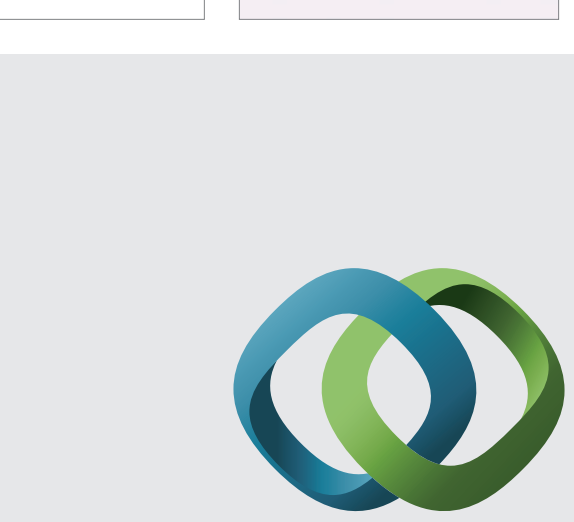

\section{Hindawi}

Submit your manuscripts at

http://www.hindawi.com
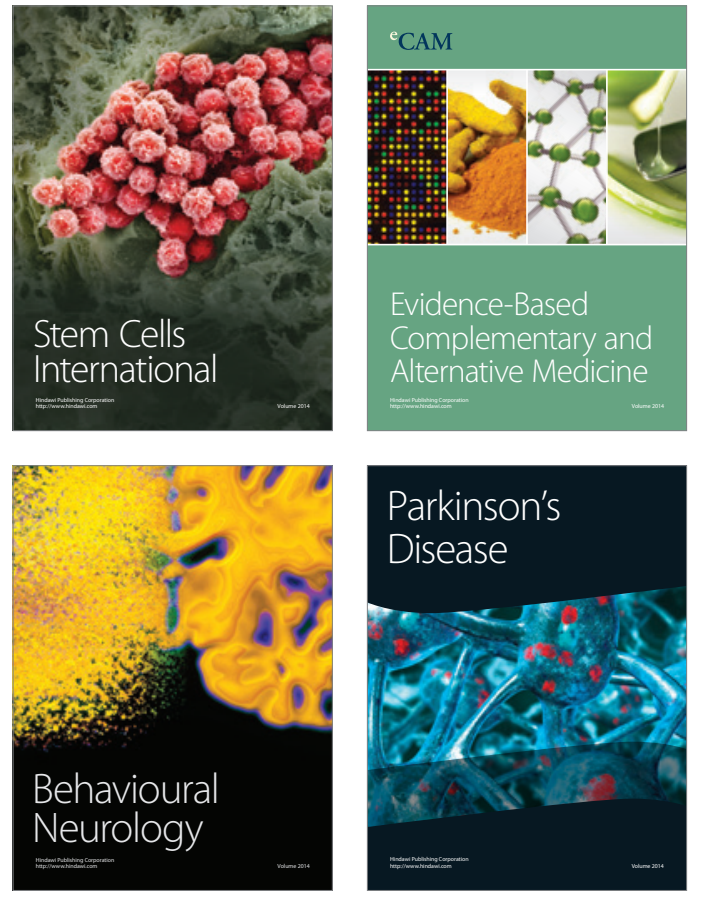
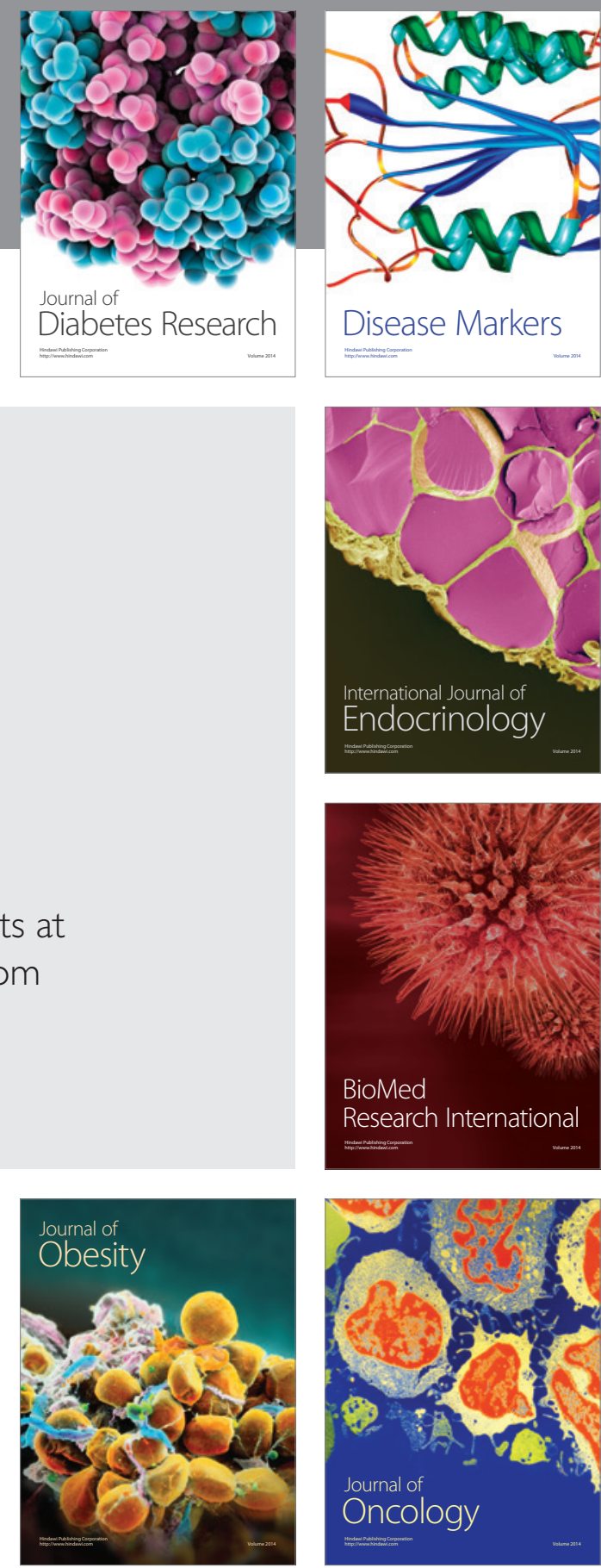

Disease Markers
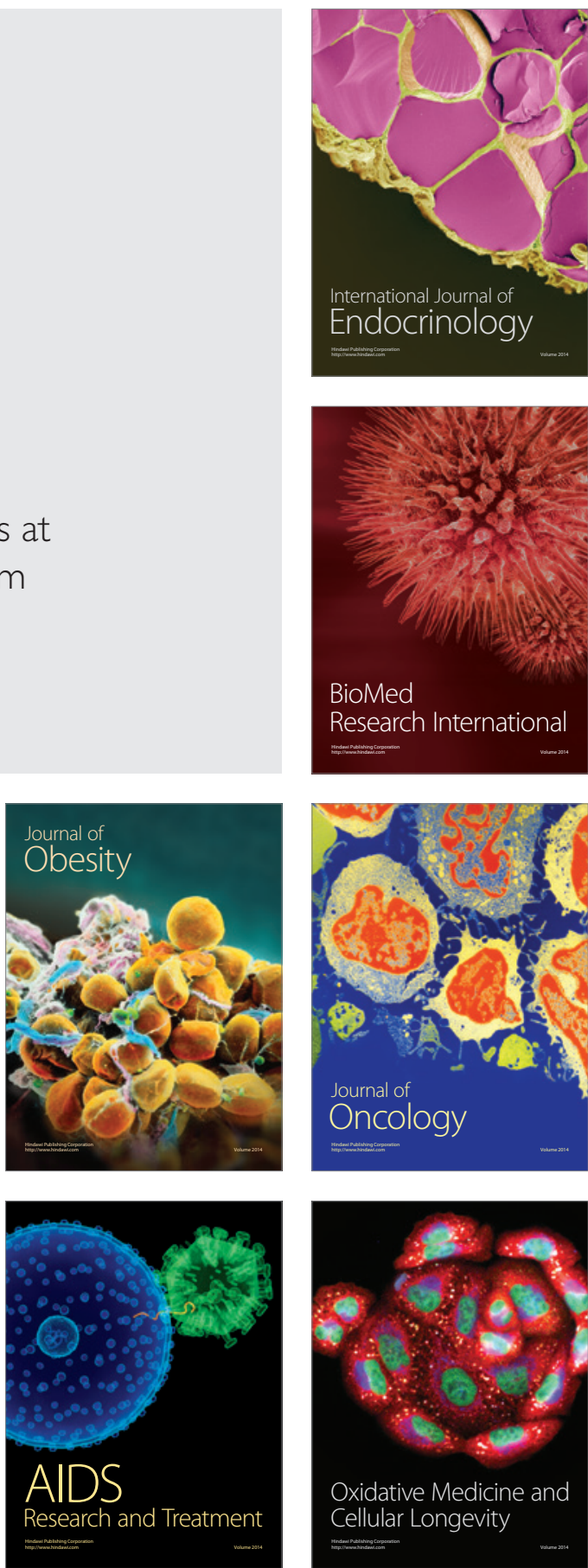